\title{
An intelligent control strategy based on ANFIS techniques in order to improve the performance of a low-cost unmanned aerial vehicle vision system
}

\author{
G. N. Marichal ${ }^{(1)}$, A. Hernández ${ }^{(1)}, M$. \\ Olivares-Méndez ${ }^{(2)}$, L. Acosta ${ }^{(1)}$, P. Campoy ${ }^{(2)}$ \\ (1) Departamento de Ing. de Sistemas y Automática, Arq. y Tec. de Comp., Universidad de La Laguna, La \\ Laguna, 38071. Tenerife. Spain. Email: nicomar@ull.es \\ (2) Computer Vision Group. Universidad Politécnica de Madrid. Madrid 28006, Spain
}

\begin{abstract}
In this paper, an intelligent control approach based on Neuro-Fuzzy systems is presented. A model of a lowcost vision platform for an unmanned aerial system is taken in the study. A simulation platform including this low-cost vision system and the influence of the helicopter vibrations over this system is shown. The intelligent control approach has been inserted in this simulation platform. Several trials taking these Neuro-Fuzzy systems as a fundamental part of the control strategy have been carried out. Satisfactory results have been achieved in comparison with the results provided by classical techniques.
\end{abstract}

\section{INTRODUCTION}

A $\mathrm{S}$ it is known vision systems onboard unmanned aerial vehicles (UAV's) are subject to highly dynamic vibrations for several sources. The vibrations produced by the engine, the rotor blades and other rotating couplings are typical examples. These vibrations introduce a significant degradation in the quality of the captured images. In fact, this degradation is a drawback in some UAV's applications. Because of that, it is often required harsh post filtering. However, it can introduce a significant phase lag, reducing undesirably the control bandwidth of the helicopter. Moreover, vibrations problems are exacerbated in small size aircrafts, where vibrations isolation is more difficult and the possible options to diminish them are limited by requirements of low weight and low power consumption. Furthermore, their cost should have a comparable scale to that of the aircraft, in order to avoid reducing the impact of such a system to market. In this paper a low-cost vision system will be considered using low quality cameras to perform the image capture. In addition to the reduction of the vibrations influence over the captured images, the motions of the dynamic platform holding the cameras should have a soft, precise and rapid response. PID controllers continue to dominate most dynamic platforms due to its simplicity and ease of design in the absence of an exact aircraft model. In this paper, it is presented an intelligent control strategy in order to improve the whole performance of a low-cost vision system.

In section II, a description of the used dynamic platform and the simulation environment developed to test the control strategies is presented. In section III, the intelligent control strategy is depicted in more detail. In fact, a description of the Adaptive Neuro-Fuzzy Inference System used to devise the intelligent control strategy is given. In section IV, some results are presented and in section $\mathrm{V}$ the conclusions are mentioned.

\section{SIMULATION PLATFORM}

In this paper the dynamic platform SPT 200 SERVICITY has been used. It is designed to support a $0.9 \mathrm{Kg}$ camera; moreover it has $1 / 4$ inch axles which are supported to double ball bearing and they make possible to put the camera on different angles of pan and tilt. There are two servomotors incorporated to the platform which allow changing the camera position according to each degree of freedom: yaw and pitch angle.

The dynamic platform mounted on the helicopter is shown in Figure 1, and the Figure 2 shows a detailed photograph of the platform and the low-cost camera.

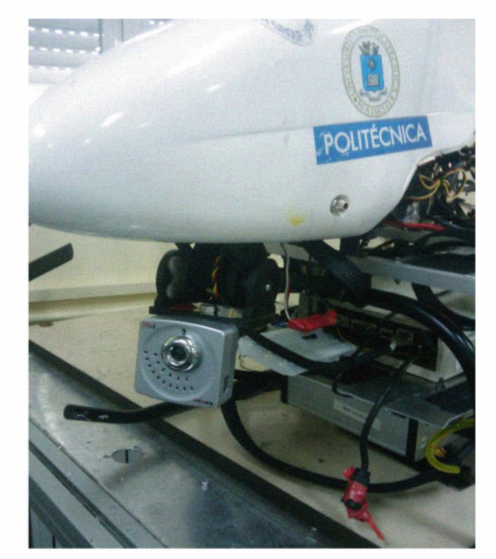

Figure 1: The platform SPT 200 mounted on the helicopter 


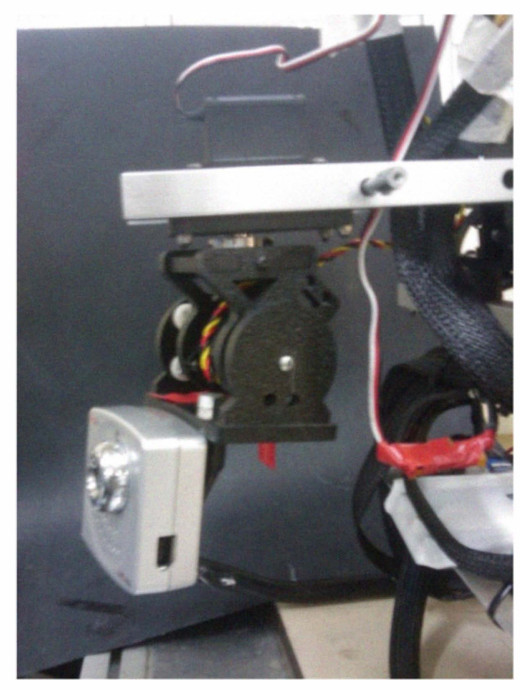

Figure 2: Photograph of the platform 1

In order to simulate this platform a detailed model has been built by a computer assisted design software. In particular the Autodesk Inventor [1] has been used in this work. The different pieces of the platform have been inserted in the model in order to achieve a detailed mechanical model. In Figure 3 it is shown the resultant model.

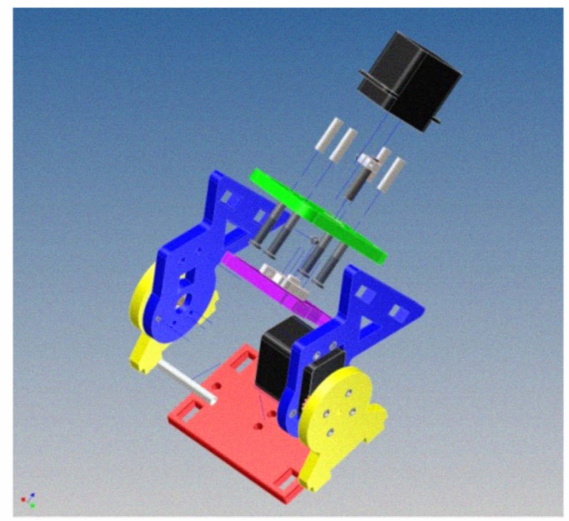

Figure 3: The SPT 200 model

The pieces have been divided into three groups: the base, the joint that allows carrying out movements on yaw angle; and the joint that allows carrying out movements on pitch angle. The inertia matrix and the centre of gravity have been calculated for each group to include them on the simulation. Furthermore, a simulation platform have been developed including additionally PID controllers for each servomotor and the camera, along with some spring-mass systems in order to take into account the flexible structure of the platform. In Figure 4 it is shown the block diagram carried out in Simulink [2].

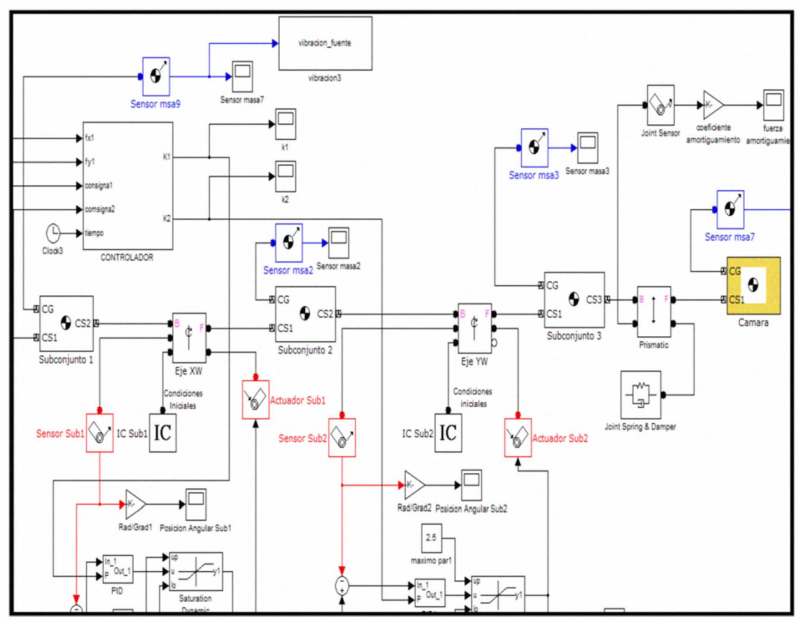

Figure 4: Some blocks of simulation platform

The model is subjected to vibrations to simulate the vibrations caused by the helicopter. Therefore two blocks were introduced to achieve a two-dimensional vibration, one of them into the axis of gravity and another into a perpendicular axis. The target of the whole system is to move the pan-tilt platform using the servomotors to certain target angles in yaw and pitch. Moreover, it is desirable when the final position is achieved the camera should be the most stable possible in order to avoid the captured image is blurred. This situation occurs if the camera position has the same coordinates $(x, y, z)$ along the time frame when the image is being captured.

It is important to remark that the performance of the control system is strongly linked to the right choice of the controller parameters. In this case, these parameters are the proportional constants $\left(\mathrm{K}_{\mathrm{p}}\right)$, derivative constants $\left(\mathrm{K}_{\mathrm{d}}\right)$ and integral constants $\left(\mathrm{K}_{\mathrm{i}}\right)$ of the two PID controllers. The equation (1) shows the final form of the PID algorithm where $u(t)$ is the controller output.

$$
u(t)=K_{p} e(t)+K_{i} \int_{0}^{t} e(\tau) d \tau+K_{d} \frac{d e}{d t}
$$

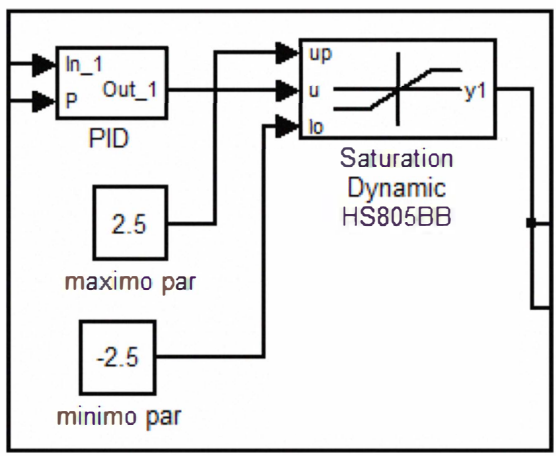

Figure 5: sketch of PID controller

The output of the PID controller indicates the torque value in order to be applied to the joints of the pan-tilt platform. 
However, there are limit values in the real situation. Because of that, this saturation condition has been modeled by a maximum and a minimum torque value as it is shown in Figure 5.

\section{CONTROL ALGORITHM}

As it was pointed out in the previous section the right election of the control parameters is essential to achieve a satisfactory result. Note that, the objectives of the control strategy are multiple. That is, it is necessary the control system allows reaching the angles setpoints in a fast and precise way. In addition to, the camera should be kept without considerable movement when the images are captured. Furthermore, the control system performance depends on the particular angle setpoints and the vibration characteristics affecting the camera position. Because of that, it is reasonable to take into consideration the particular angle setpoints and the particular vibrations affecting the camera movements in order to choose the controller parameters. In this paper, a criterion function has been defined in order to associate a performance index with each setpoint and each kind of vibration. Furthermore, their frequencies have been taken as significant characteristic of the vibrations. The criterion function has been defined such that it is weighted the displacement of the camera around the final position, the pitch angle error and the yaw angle error. In this way, the values of the criterion function are directly linked to the performance of the control system in terms of precision in the desired angles and small movements when the images are being captured. In fact, a value of the criterion function could be calculated for each combination of pitch angle setpoint, yaw angle setpoint, frequency of vibration in the gravity axis $\left(f_{y}\right)$ and frequency of vibration in the perpendicular axis $\left(f_{x}\right)$, in addition to a particular selection of the controller parameters. In fact, only sinusoidal vibrations have been considered in order to evaluate the criterion function. Taking into account these dependencies an iterative process were carried out where the proportional constants of both PID controllers were modified, keeping other parameters invariant for each combination of pitch angle setpoint, yaw angle setpoint, frequency of vibration in the gravity axis and frequency of vibration in the perpendicular axis. This approach has been used by simplicity, given a modification of more controller parameters will contribute to increase in a notably way the computer charge of the iterative process The proportional constant were modified between 5 and 55 in steps of 10 units for each combination. Once the iterative process has been finished it was possible to obtain the proportional constants, which minimize the criterion function for each combination of pitch angle setpoint, yaw angle setpoint, frequency of vibration in the gravity axis and frequency of vibration in the perpendicular axis. In this way, it is obtained a matrix with 10080 rows, considering in each row the proportional constants with a minimum value of the criterion function and the combination of values indicated above. In this calculation process yaw values from 0 to $90^{\circ}$, pitch values from $10^{\circ}$ to $90^{\circ}$, frequencies on the axis of gravity from 1 to $131 \mathrm{~Hz}$, and frequencies on the perpendicular axis from 1 to $71 \mathrm{~Hz}$ have been considered. It is important to point out that the typical vibration frequencies are around this range [3] [4]

At this point, it would be adequate to devise a system, which is able to provide the most convenient proportional constants for each situation. The Artificial intelligence techniques based on a training set could be a good choice. One option could be the Neural Networks [5] [6], where their learning properties are adequate for the problem undertaken. However, the Neural Network is essentially a 'black box'. Because of that, it has often been criticized for exhibiting a low degree of human comprehensibility. Determining exactly why it makes a particular decision is a daunting task. Instead of, an alternative Neuro-Fuzzy approach has been chosen in this paper. A Neuro-Fuzzy approach based on the scheme proposed by Jang [7] [8] [9] has been used. This kind of approaches is known as Adaptive Neuro-Fuzzy Inference Systems (ANFIS systems). In this framework, a neuro-adaptive learning method has been used. This method works similarly to neural networks, using a given input/output data set.

It is a method that interprets the values in the input vector based on a set of rules and assigns values to the output vector. This involves the choice of the membership functions and fuzzy logics operators, the design of fuzzy rules, the choice of the aggregation mechanism, the involvement of the fuzzy rules (inference mechanism), and finally, the defuzzification method for obtaining a numeric output. In Figure 6 it is shown a diagram of the used ANFIS.

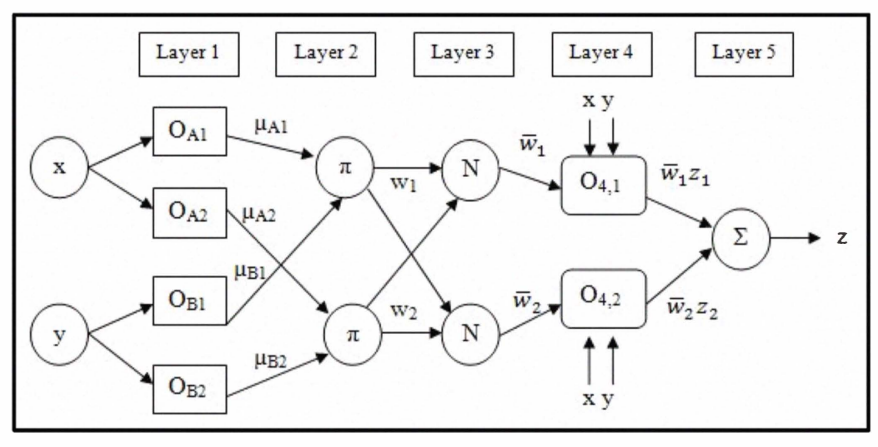

Figure 6: ANFIS architecture

In the following a description of the ANFIS structure is presented. For the sake of simplicity, the structure will be particularized to the case of a system with two inputs, $x$ and $\mathrm{y}$, each one with two membership functions, $\mu \mathrm{Ai}$ y $\mu \mathrm{Bj}$, respectively, and an output $z$. Hence, this system is associated with two fuzzy if-then rules of Takagi-Sugeno type which are: 
If $\mathrm{x}$ is $\mu_{\mathrm{A} 1}$ and $\mathrm{y}$ is $\mu_{\mathrm{B} 1}$ then $\mathrm{z} 1=\mathrm{p} 1 \mathrm{x}+\mathrm{q} 1 \mathrm{y}+\mathrm{r} 1$;

If $\mathrm{x}$ is $\mu_{\mathrm{A} 2}$ and $\mathrm{y}$ is $\mu_{\mathrm{B} 2}$ then $\mathrm{z} 2=\mathrm{p} 2 \mathrm{x}+\mathrm{q} 2 \mathrm{y}+\mathrm{r} 2$;

where $\mathrm{p}_{\mathrm{i}}, \mathrm{q}_{\mathrm{i}}, \mathrm{r}_{\mathrm{i}}$ are the consequent parameters.

In the first layer, each node has an output defined as:

$$
\begin{gathered}
O_{A, i}=\mu_{A i}(x) \\
O_{B, i}=\mu_{B i}(y), i=1, \ldots, n
\end{gathered}
$$

where $n$ is the number of membership functions of the inputs $\mathrm{x}$ and $\mathrm{y}$, because, in this case, it is assumed that the two inputs have the same number of membership functions.

The second layer multiplies the input signals and each output of a node $\pi$ corresponds to the consequent for each rule. Note that, it represents the weight of the conclusion of each rule:

$$
w_{i}=\mu_{A i}(x) \mu_{B i}(y), i=1, \ldots, n
$$

In the third layer, the output of each node $\mathrm{N}$ corresponds to the standard weights:

$$
\overline{w_{l}}=\frac{w_{i}}{\sum_{i=1}^{n} w_{i}}, i=1, \ldots n
$$

The fourth layer calculates the output as a sum of the previous ones:

$$
O_{4, i}=\bar{w}_{i} z_{i}=\bar{w}_{i}\left(p_{i} x+q_{i} y+r_{i}\right), i=1, \ldots, n
$$

Finally, the fifth one adds all outputs of the fourth layer and it gives as output a real number:

$$
O_{5, i}=\sum_{i} \bar{w}_{i} z_{i}, i=1, \ldots, n
$$

It could be said that the ANFIS systems can be broken down into three main phases: collection of input/output data in a form that it will be usable by ANFIS for training, the creation of a Fuzzy System [10] as initial structure, and the application of a learning algorithm consisting of a combination of the least-squares method and the backpropagation gradient descent method for training the ANFIS parameters. It is important to remark that these parameters are the premise and consequent parameters. In this paper, the input to the ANFIS system are the pitch setpoint, the yaw setpoint, the frequency of the vibration in the gravity axis and the frequency of the vibration in the perpendicular axis, whereas the outputs are the proportional constants for the PID controllers in the yaw and pitch degree of freedom.

Once we have chosen the ANFIS structure described previously, the ANFIS was trained with the eighty percentage of the data obtained above. Once the training phase has been concluded the twenty percentage of data are used to validate the ANFIS system. That is, a certain number of data are reserved to test the generalization properties of the resultant ANFIS. Note that, the final system is a zeroorder Sugeno fuzzy inference system [11]. In fact, it is important to remark that two ANFIS systems with one output were used, instead of one ANFIS system with two outputs. In this way, a better behavior of the resultant ANFIS systems was achieved.

This two trained ANFIS systems are incorporated to the simulation environment into two simulink blocks (ANFIS $\mathrm{K}_{\mathrm{p} 1}$ and ANFIS $\mathrm{K}_{\mathrm{p} 2}$ ). These blocks will be referred as ANFIS blocks in the following. The ANFIS blocks need as input the desired yaw and pitch angles and the frequencies of the vibration signals. In figure 7 it is shown a sketch with the ANFIS blocks.

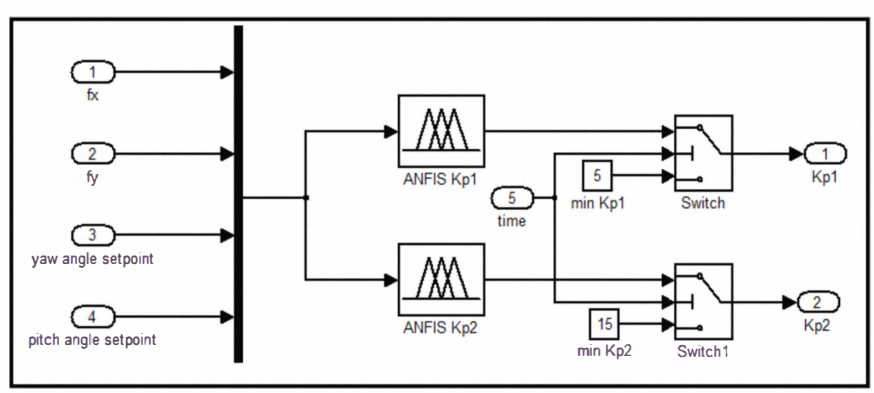

Figure 7: ANFIS controller blocks.

The desired yaw and pitch angles are marked by the trajectory to follow, whereas the frequencies are determined by other blocks. These blocks have been introduced between the vibration signal and the ANFIS Controller blocks. Note that, the vibration signals applied in this case have a certain spectral composition of frequencies. They are not sinusoidal signals in general. Because of that, these blocks are in charge to apply the Fast Fourier transform and to obtain a certain number of dominant frequencies.

Figure 8 shows as the vibration signals are introduced in the FFT blocks in order to calculate the frequencies each 0.5 seconds.

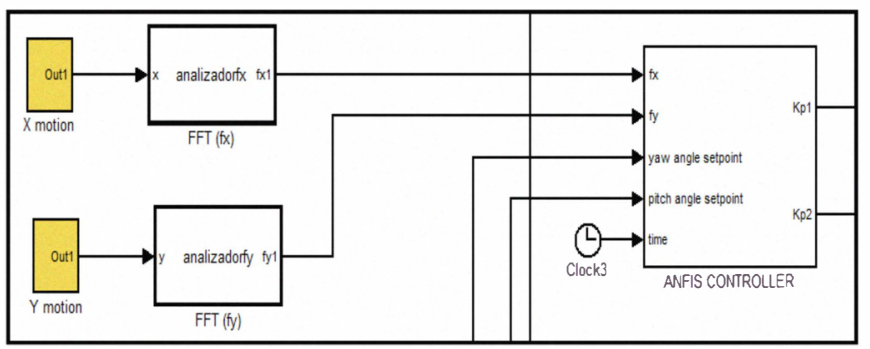

Figure 8: FFT blocks

In this paper, ten dominant frequencies have been taken for each vibration signal. That is, the first ten frequencies 
sorted by the magnitude of the FFT components are considered, and they are sent to the ANFIS Controller blocks. Note that, there are ten dominant frequencies for $f_{x}$ and fy respectively. These frequencies are considered in the system shown in Figure 7, obtaining a $K_{p 1}$ and a $K_{p 2}$ for each frequency. After that, a weighted average is calculated in order to achieve the final proportional constant of the respective PID controllers. In this case, the weights are the different multiplications of the amplitudes corresponding to the dominant frequencies of $f_{x}$ and fy. As it can be seen in figure 7, two initial proportional constant values are taken for the first 0.5 seconds. After, the proportional constant values are chosen as it was pointed out above.

In figure 9 it is shown the frequency of the maximum amplitude in the perpendicular axis versus the time from one of the FFT blocks.

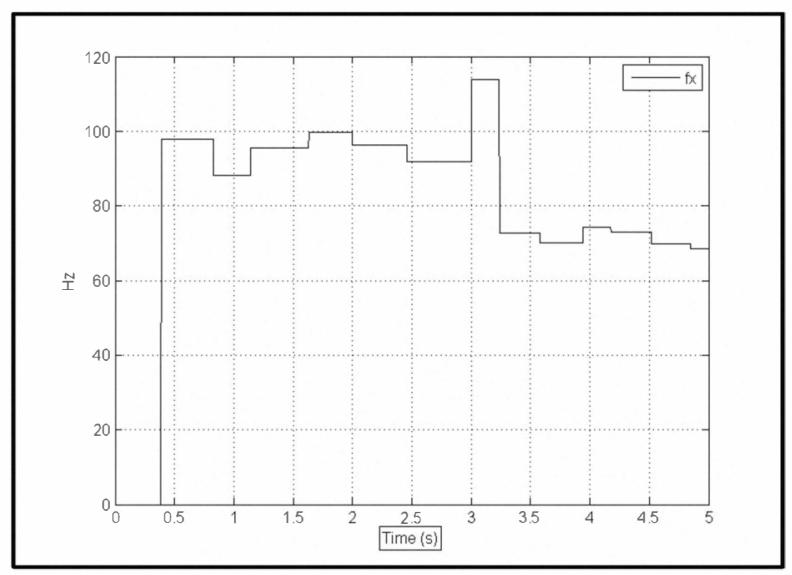

Figure 9: Variations of the frequency of maximum amplitude in the perpendicular axis versus the time. It was obtained by the FFT of the vibration signal for each $0.5 \mathrm{~s}$.

In summary, it could be said that these different frequencies are used as inputs to the ANFIS system and a weighted average is applied to the obtained outputs, where the weights are the magnitudes of the respective FFT components. In this way, it is obtained a value for the proportional constant $\mathrm{K}_{\mathrm{p} 1}$ to the PID controller linked to the yaw degree of freedom and another value $K_{p 2}$ for the PID controller linked to the pitch degree of freedom. Note that this is the core of the intelligent control strategy and that the accelerations of the vibrations are directly measured by an accelerometer sensor before being processed.

IV. APPLICATION

Once the intelligent control strategy has been depicted in the previous section, along with the simulation platform the results of the trials are shown in this section. Several trials have been carried out. In these trials as it was pointed out in the previous section desired yaw values from 0 to $90^{\circ}$ and pitch values from 10 to $90^{\circ}$ have been considered. On the other hand, the possible frequencies of the vibration on the axis of gravity from 1 to $131 \mathrm{~Hz}$, and frequencies on the perpendicular axis from 1 to $71 \mathrm{~Hz}$ have been considered. This range of vibrations is typical in small helicopters, considering that the most influence vibrations are originated in the engine, the main rotor and the tail rotor.

In order to test the algorithm several trajectories of yaw and pitch angles have been tested. In figure 10 it is shown the evolution of the coordinate $\mathrm{x}$ with the intelligent control strategy versus the evolution using only the classical PID technique.

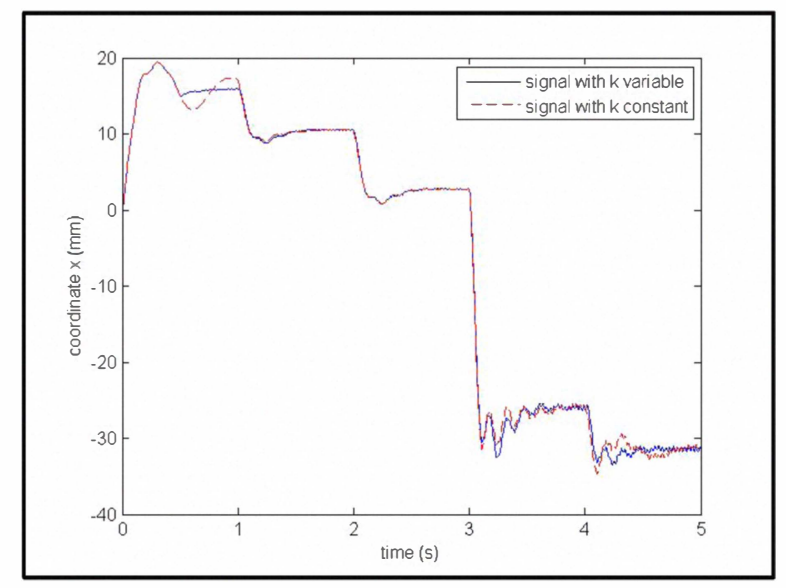

Figure 10: Comparison between the evolution of the coordinate $\mathrm{x}$ if the proportional parameter is variable or fixed

In this case, the yaw setpoint angles and pitch setpoint angles have been kept invariant during intervals of one second. As it could be appreciated in figure 10 the coordinate $\mathrm{x}$ is practically constant at one second and in the period of time near one second with a changeable proportional constant. Whereas, when a fixed proportional constant is taken there is a considerable displacement. However, the responses are similar at 2, 3 and 4 seconds. In the case of the period between 4 and 5 seconds small differences are appreciated. It is important to point out that the intelligent control approach has got a better behaviour in order to capture images with a better quality. Similar results are obtained for the other coordinates.

In order to study the influence of the control algorithm in the evolution of the angles, a graph of the yaw angle versus time is shown in Figure 11.

As it could be appreciated considerable better results are achieved for the intelligent control approach in the interval between 0 and 1 second. In the case of the others intervals, similar results are obtained. However, it is important to remark that a softer response is obtained by the intelligent control approach and the angle setpoint is reached before. Similar results are obtained for the pitch angle.

In figure 12 the changes in the proportional constant for the PID linked to the yaw angle is shown. As it can be seen there is an abrupt jump at 0.5 seconds. It is because there are not frequencies values before this time. That is, the 
algorithm waits for a small period of time in order to process the accelerations in order to obtain the spectral frequency. Because of that, it is taken a fixed initial proportional constant. Once 0.5 seconds have been elapsed the intelligent control approach starts giving proportional constant values according to the outputs of ANFIS systems.

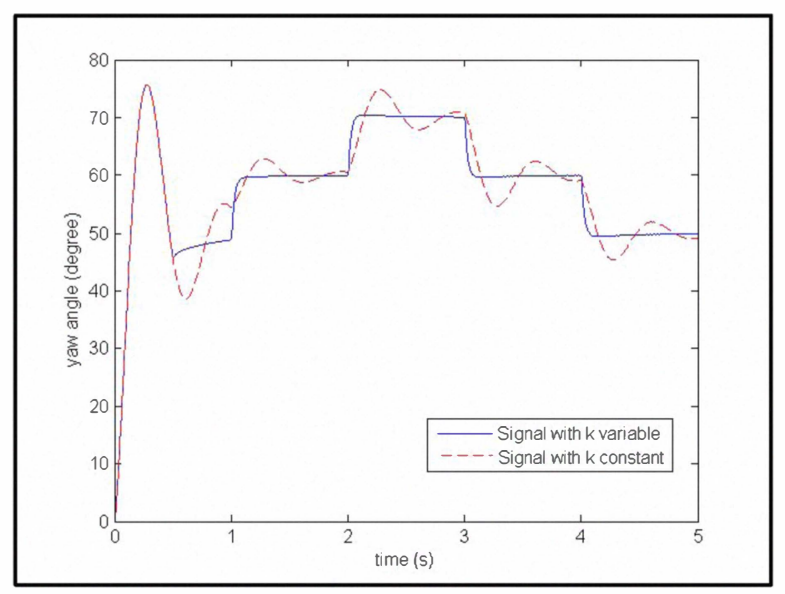

Figure 11: Comparison between the movements occurred on the yaw angle if the proportional parameter is variable or fixed

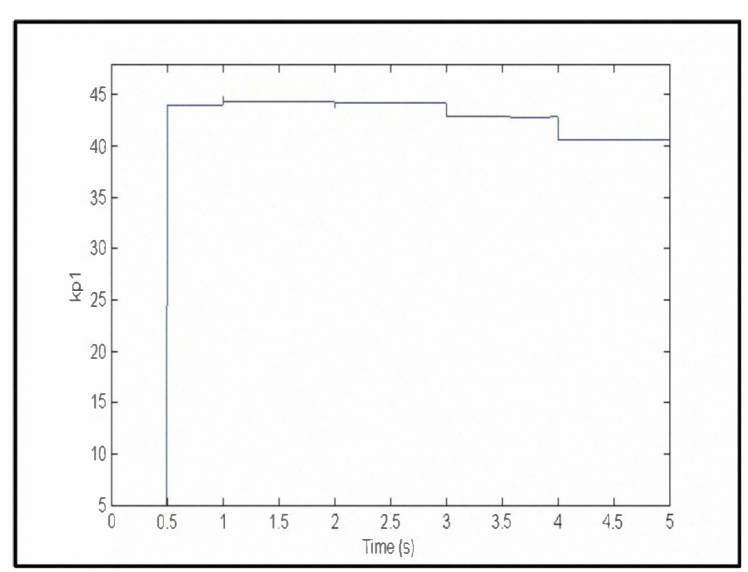

Figure 12: Variations of $\mathrm{kp} 1$ with the time

\section{CONCLUSION}

In this paper a simulation platform of a low-cost vision system for an unmanned aerial vehicle has been built. This platform allows simulating the influence of the typical helicopter vibrations and the motion of the pan-tilt platform. A detailed model of this platform has been incorporated to the global simulation platform. Furthermore, an intelligent control strategy based on ANFIS systems has been devised and tested. Satisfactory results have been achieved in the carried out trials.

\section{ACKNOWLEDGMENT}

We would like to thank the financial support provided by
Spanish government projects DPI2007-66156 in collaboration with Polytechnic University of Madrid and DPI2007-64137.

\section{REFERENCES}

[1] Autodesk ${ }^{\circledR}$ Inventor ${ }^{\mathrm{TM}}$. Available online: http://www.autodesk.com/inventor/.

[2] Simulink ${ }^{\circledR}$ Available online http://www.mathworks.com/products/simulink/

[3] Matthew Dunbabin, Stephen Brosnan, Jonathan Roberts, and Peter Corke. Vibration isolation for autonomous helicopter flight. In Proceedings of the International Conference on Robotics and Automation,

[4] G. Buskey, J. Roberts, P. Corke, M. Dunbabin and G. Wyeth. The CSIRO autonomous helicopter project. In Proceeding of the International Symposium on Experimental Robotics, 2002

[5] B. Kosko. Neural Networks and Fuzzy Systems: A Dynamical Systems Approach to Machine Intelligence, Prentice-Hall, Englewood Cliffs, NJ (1992).

[6] Pajares, G. A Hopfield Neural Network for Image Change Detection. IEEE Trans. Neural Netw. 2006, 17, 1250-1264

[7] Jang, J.R. ANFIS: Adaptive-network-based fuzzy inference system. IEEE Trans. Syst. Man Cybern. 1993, 23, 665-685

[8] Mitra, S; Hayashi, S. Neuro-fuzzy rule generation: Survey in soft computing framework. IEEE Trans. Neural Netw. 2000, 11, 748-768..

[9] Marichal, G.N.; Acosta, L.; Moreno, L.; Mendez, J.A.; Rodrigo, J.J.; Sigut, M. Obstacle avoidance for a mobile robot: a neuro fuzzy approach. Fuzzy Sets and Systems. 2001, vol. 124, pp. 171-179.

[10] Zadeh, L.A. Knowledge representation in fuzzy logic. IEEE Trans. Knowl. Data Engin. 1989, 1,89-100.

[11] Ying, H. General Takagi-Sugeno fuzzy systems are universal approximators. In Proceedings of the IEEE International Conference on Fuzzy Systems, Anchorage, Alaska, May 4-9, 1998.

[12] G. Buskey, J. Roberts, P. Corke, G. Wyeth,Helicopter Automation using Low-Cost Sensing System, Australian Conference on Robotics and Automation, 2003

[13] Gavrilets, V., A. Shterenberg, M.A. Dahleh, E. Feron, “Avionics system for a small unmanned helicopter performing aggressive maneuvers," Proceedings of the 19th Digital Avionics Systems Conferences,

[14] Sugeno, M., I. Hirano, S. Nakamura, S. Kotsu, "Development of an intelligent unmanned helicopter," Proceedings of 1995 IEEE International Conference on Fuzzy Systems, pp. 33-34, Yokohama, Japan, 1995

[15] J.F Montgomery and A. Bekey. Learning helicopter control through "teaching by showing". IEEE Conference on Decision and Control, 4:3647 - 3652, 1998

[16] Eisenbeiss, H., 2004. A mini unmanned aerial vehicle (UAV): system overview and image acquisition. International Archives of Photogrammetry. Remote Sensing and Spatial Information Sciences. $36(5 / \mathrm{W} 1)$

[17] Nakamura, S., Kataoka, K. \& Sugeno, M. (2001). A Study on Autonomous Landing of an Unmanned Helicopter Using Active Vision and GPS. The Journal of Robotics Society Japan, Vol. 18, No. 2, pp. 252-260

[18] Saripalli, S., Montgomery, J.F. \& Sukhatme, G. S. (2003). Visually-guided landing of an unmanned aerial vehicle. IEEE Transactions on Robotics and Automation, Vol. 19, No. 3, pp. 371-381

[19] Shakernia, O., Ma, Y., Koo J. \& Sastry, S.S. (1999). Landing an Unmanned Aerial Vehicle: Vision Based Motion Estimation and Nonlinear Control. Asian Journal of Control, Vol. 1, No. 3, pp. 128-145.

[20] R. Enns and J. Si. Helicopter tracking control using direct neural dynamic programming. Proceedings of the International Joint Conference on Neural Networks, 2:1019-1024, July 2001.

[21] .A. Bagnell and J.G. Schneider. Autonomous helicopter control using reinforcement learning policy search methods. In International Conference on Robotics and Automation, 2001

[22] G. Buskey, J. Roberts, and G. Wyeth. Online learning of autonomous helicopter control. In Australasian Conference on Robotics and Automation, Auckland, New Zealand., December 2002 